\title{
A Study of Human's Horoscope Based on Fractals and Analyzation of Planet's Consistency
}

\author{
R. Kamali ${ }^{1}$, G. Jayalalitha ${ }^{2}$ \\ ${ }^{I}$ Research Scholar, Department of Mathematics, VELS Institute of Science, Technology and Advanced Studies, \\ Chennai-600 117,Tamil Nadu, India. \\ E-mail: kamali_1883@yahoo.co.in \\ ${ }^{2}$ Professor, Department of Mathematics, VELS Institute of Science, Technology and Advanced Studies, \\ Chennai-600 117, Tamil Nadu, India. \\ E-mail: ragaji94@yahoo.com
}

\begin{abstract}
The aim of this paper is to study the human's Horoscope by using Fractals, a mathematical analysis to solve a high degree of geometrical complexity. As the universe is complex the structure of the Astrological aspects can be analyzed by Fractal dimensions using the method Diffusion depending upon the time complexity. The Random walk is also observed for a certain period of time based on Diffusion of the planets in the Horoscope. The variations in the irregular combinations of the planets in the Horoscopes are found using Coefficient of Variation.
\end{abstract}

Keywords: Coefficient of Variation, Diffusion, Fractals, Horoscope, Random walk.

\section{INTRODUCTION}

A Horoscope is an Astrological chart or diagrammatic representation of the positions of the Sun, the Moon, the planets, astrological aspects and sensitive angles at the time of a person's birth (Svenson et al., 1995). The word horoscope is derived from Greek words hõra and scopos meaning "time" and "observer". There are 12 zodiac signs each of it has its own strength and weakness, its own specific traits, desires and attitude towards life and people (Ronny Martens et al., 1998). Each of these 12 signs belongs to a certain element in one of its states. These varying qualities provide the circumstances to the planetary positions. Each horoscope has different planets in different signs; so each horoscope is a highly individual, very complex and also very varied combination of parts (Ove $\mathrm{H}$. Sehested, 1973). By analyzing the projection of the position of planets, the Sun and the Moon on the ecliptic at the moment of birth; Astrologers can give us a glimpse of a person's basic characteristics, preferences, flaws and uncertainties. The different Horoscopes mentioned in Fig. 1 are the South Indian style birth chart which shows the 12 Rasi and the position of planetary bodies in each Rasi.
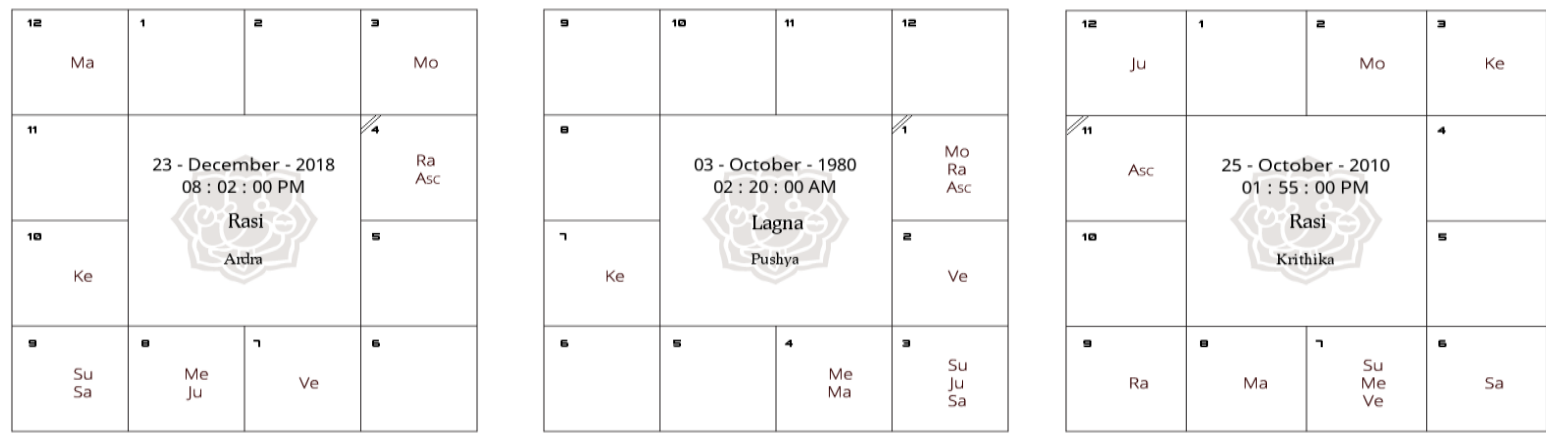

Figure 1: Model of Human's Horoscope

Basically the Horoscope is divided into twelve sections referred to as houses. Each house represents an area of life. The houses one to six address everyday activities, finances, etc. and the houses seven to twelve relates to more abstract concepts. The unique placement of the planets in the houses is determined by the rising sign, also known as the ascendant. This is the zodiac sign that was on the eastern horizon at the exact moment of your birth.
Depending upon the placement of all the planets in the different houses in a horoscope leads to an irregular combination which looks like a Fractal structure; Fractals are introduced in order to describe the irregular and the fragmented patterns of nature in a sufficient way (K. Falconer, 1997). An important property of Fractals is their fractal dimension. The planets fractal dimension can be used as a tool to predict the future. The distance and the angle between 
the planets can be approximated by Fractal geometry (Falconer K, 2014).

Fractal geometry provides methods for characterizing the complexity and the consequent position of the planets in one's Horoscope, since placement of the planets are irregular (M.T. Barlow, 1998). One among them is Diffusion; this processes can be found everywhere in our daily life showing different time scaling behaviour in which the pattern of the planets placement and its complexity are analysed. The Fractal dimension is an important quantity to characterize diffusion properties (R. Metzler et al., 2000). A Conjunction is formed among the planets which are located close together. Planets occupying the same degree of the same sign will be in exact Conjunction. Generally planets in Conjunction are powerfully united and their energies merge. If the planets involved in the conjunction are benefic or one benefic and one malefic the effect of the conjunction is usually favourable, but if the planets are malefic the effect is usually unfavourable. This results in the irregularity in the combination of planets and their effects. Observing such variations for a period of time; it is probable to find that the planets are performing a Random walk. A simple model to put forward diffusion is the random walk method too (J.M. Angulo et al., 2000). A Random walk is nothing but a fundamental physical process in which a random walker i.e., a particle or an individual moves in random steps (Bunde A et al., 1996 and Uthayakumar $\mathrm{R}$ et al., 2009).This can be illustrated using graphs which gives the clear vision of the planet's motion in one's horoscope. The Coefficient of variation (S.P. Gupta, 2007) is applied to the positions of the planets to classify their effectives in the Horoscope which is very useful for predicting the future of a person.

In section 2 the Diffusion process and the Random walk for the planets is explained in detail. In section 3 the consistency of the planets position is discussed with a suitable example.

\section{METHODS}

The methods involved in this paper are as follows:
In astrology, the situation in which two planets appear to be in the same part of the sky as seen from earth is the conjuncture, since a conjunction of two or more things is the occurrence of them at the same time or place. The condition of two or more celestial bodies, i.e. a planet with the sun, located along the same celestial longitude when observed from the earth seems to form a Diffusion among the planets. Diffusion process is mainly taken place based on time scales. Time to time, there is an irregular change in the position of the planets as they are under revolution. Thus the placement of the planets and the combination of the planets are irregular in the horoscopes for different persons. This irregularity is analysed by the Diffusion process. Basically the Diffusion process is modelled by a simple random way which in one time unit advances one step of length ' $a$ ' to a randomly chosen nearest-neighbour site on a given $d$-dimensional pattern. Assume that a random walker starts at time $t=0$ at the origin of the pattern. After $t$ time steps, the actual position of the process is described by the vector

$a \sum_{\tau=1}^{t} e_{\tau}$

$r(\mathrm{t})=$

where $e_{\tau}$ denotes the unit vector pointing in the direction of the jump at the $\tau t h$ time step.

The average distance formed between the planets due to the rotation of the earth after ' $t$ ' time steps is described by the root mean square displacement $\left\langle r^{2}(t)\right\rangle^{1 / 2}$, where the average $\langle\ldots\rangle$ is over all random way configurations of the planets in the pattern and it obtains from Fick's law of diffusion

$a^{2} t$

$$
\left\langle r^{2}(t)\right\rangle=
$$

eqn (2) is independent of the dimension $d$ of the pattern.

\section{Diffusion Process}

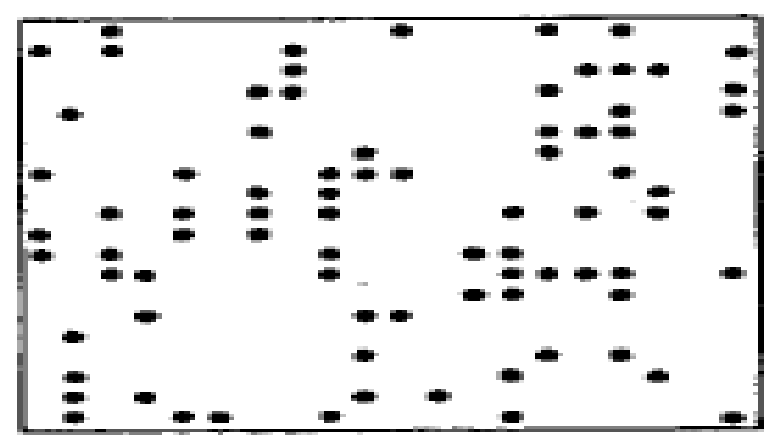

Figure 2: Diffusion of Planets 


\section{Available online at www.ijrat.org}

To characterize the diffusion for the planets on regular as well as random way as in Fic 2 , the longest decay time of the probability distribution, the mean exit time and the Randon walk dimension can be determined. In general when the planets has a probability to stay in place

$2 d \mathcal{D} t$

$$
\left\langle r^{2}(t)\right\rangle=
$$

where $\mathcal{D}$ is the diffusion constant. The mean square displacement can be obtained from the probability density $P(r, t)$, which is the probability of finding the planet after $t$ time steps at a site within distance $r$ from its starting point, via

$$
\int r^{2} P(r, t) d r
$$

$$
\left\langle r^{2}(t)\right\rangle=
$$

After $t$ time steps, the planet has a movement $m$ times to the right and $t-m$ times to the left, so its actual position is at $x$, is described by the binomial distribution

$$
P(m, t)=\left(\begin{array}{c}
t \\
m
\end{array}\right) P^{m}(1-p)^{1-m}
$$

$\left(\begin{array}{c}t \\ m\end{array}\right)\left(\frac{1}{2}\right)^{\prime}$

For large $t$ the binomial coefficient can be expressed by the Stirling's formula, $t ! \cong(2 \pi t)^{1 / 2}(t / e)^{t}$. When the planet has moved a neutral distance $x$ from the starting point, it acquires

$$
\frac{1}{(2 \pi t)^{1 / 2}} e^{-x^{2} / 2 t}
$$

$$
P(x, t) \cong
$$

Since $P(x, t)$ represents a probability, $\int_{-\infty}^{\infty} P(x, t) d x=1$, which is satisfied by (6). In a more general case of a $d$-dimensional hypercubic pattern, eqn (6) simply becomes

$\frac{1}{\left\langle r^{2}(t)\right\rangle^{d / 2}} e^{-(d / 2) r^{2} /\left\langle r^{2}(t)\right\rangle}$

$$
P(r, t)=
$$

where $\left\langle r^{2}(t)\right\rangle=2 d \mathcal{D} t$ and the probability $P(0, t)$ that the planet is at the origin after $t$ time steps is proportional to $\left\langle r^{2}(t)\right\rangle^{-d / 2}$.

In this paper it is assumed that the time and space to be continuous. The focus is on Diffusion within a certain finite area with absorbing boundaries. It determines the probability distribution of particles for each time step. The limitation of Diffusion is also allowed to undergo a random walk due to Brownian motion; cluster together to form aggregates of such planets combination in one's Horoscope. To identify with Diffusion in this combination of planets, the selfsimilarity and complexity have to be captured in the space model.

\section{Random Walk}

Furthermore, the astrological aspects are used to determine the angular relationships between the various celestial bodies and angles in the horoscope. In one dimension a Random walk can be described by its random individual steps, $e_{i}$, where $i$ is the step number. The step $e_{i}$ is a random variable that may have some distribution as in Fig 3. It is possible to express Random walk on a graph by the process of visiting the planets of the graph in some sequential random order. This movement starts at some fixed planet and at each step it moves to a neighbor of the current planet chosen randomly.

Figure 3: A random walk of the planets in a square lattice

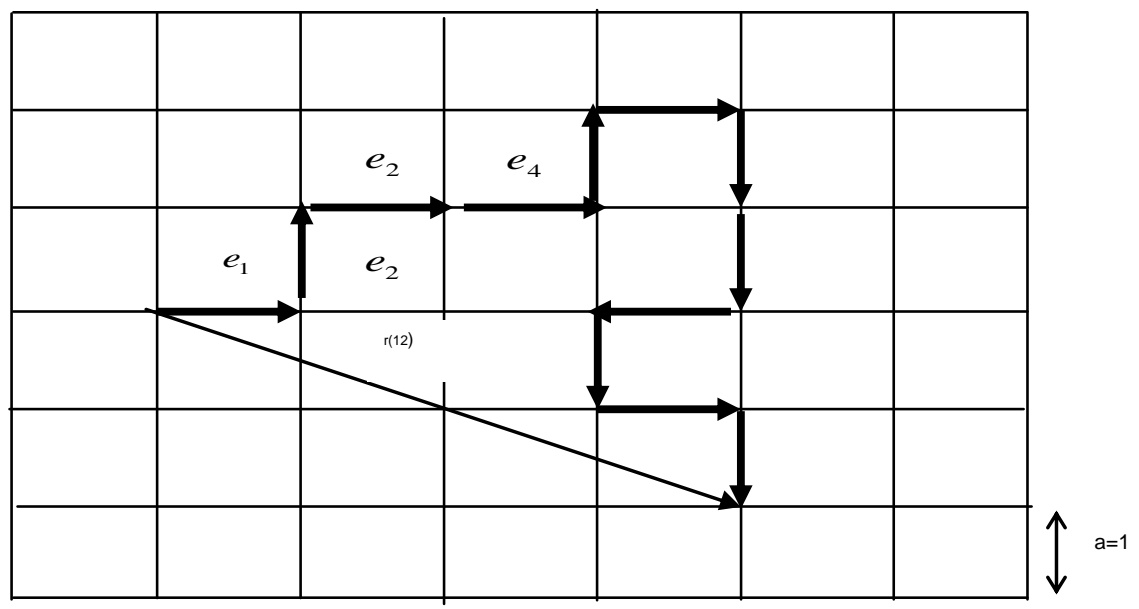




\section{Available online at www.ijrat.org}

The Random walk is simple because the next planet is chosen uniformly at random from the set of neighbors. This Random walk model can be mapped directly onto the Diffusion equation since the motion of the planets depends on his previous trajectory.

Considering for example, the Horoscopes of four different persons as in Table 1 in which the exact position of the planets is mentioned clearly.
This progress of the planets can be captured apparently by Random walk using graphs. These Random walks of the planets are entirely different for each person's Horoscope as shown in Fig 4 and Fig 5. Hence there is an irregularity among the motion of planets in each person's Horoscope this leads to the formation of Fractals in the Horoscopes.

Table 1: Positions of the 9 planets in 4 different persons Horoscopes

\begin{tabular}{|c|c|c|c|c|}
\hline \multirow{2}{*}{ PLANETS } & \multicolumn{4}{|c|}{ POSITIONS OF THE PLANETS IN THE HOROSCOPE } \\
\cline { 2 - 5 } & Person1 & Person2 & Person3 & Person4 \\
\hline 1 & 324.56 & 31.41 & 65.86 & 305.14 \\
\hline 2 & 50.85 & 117.9 & 187.37 & 118.74 \\
\hline 3 & 307.58 & 200.33 & 213.59 & 67.98 \\
\hline 4 & 37.22 & 107.58 & 192.58 & 105.1 \\
\hline 5 & 121.55 & 113.45 & 330.51 & 205.94 \\
\hline 6 & 75.45 & 139.74 & 193.96 & 113.53 \\
\hline 7 & 215.15 & 348.68 & 166.64 & 110.58 \\
\hline 8 & 224.59 & 7.96 & 251.15 & 35.03 \\
\hline 9 & 44.59 & 187.96 & 71.89 & 215.03 \\
\hline
\end{tabular}

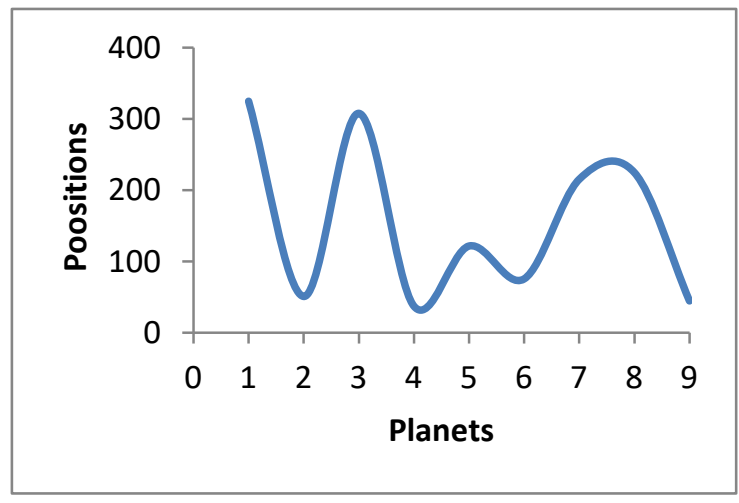

Horoscope (i)

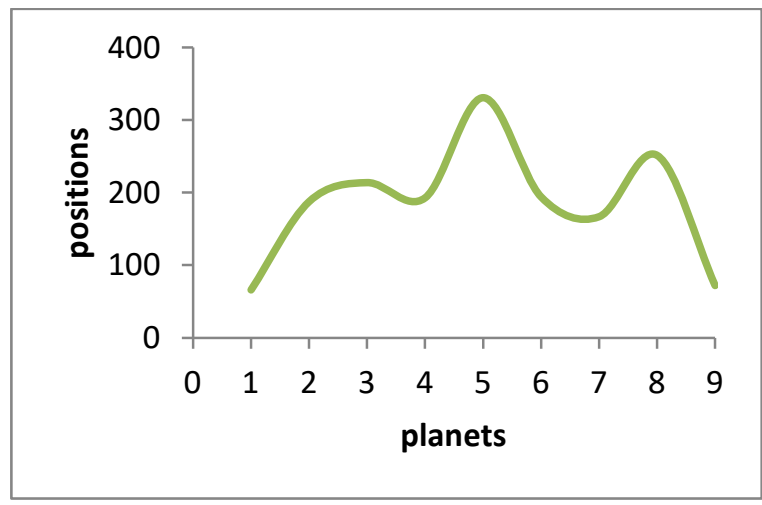

Horoscope (iii)

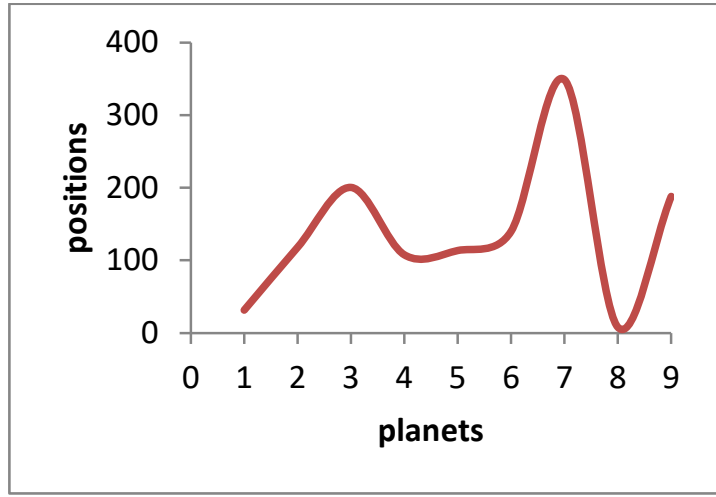

Horoscope (ii)

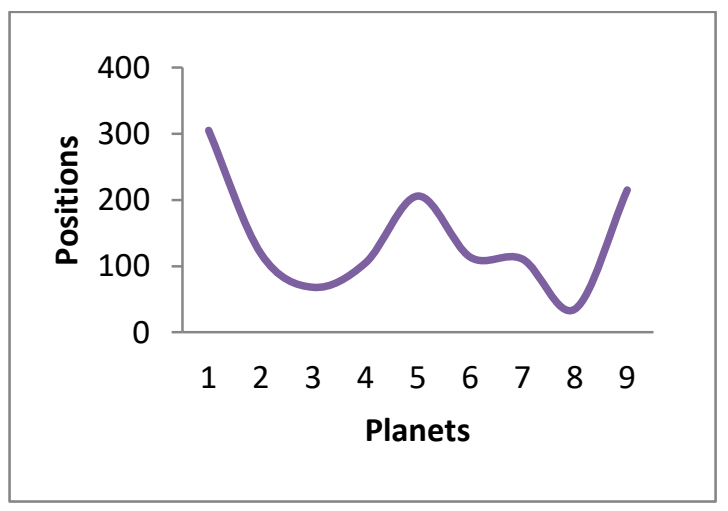

Horoscope (iv)

Figure 5: Random Walk in 4 different person's Horoscopes 


\section{Available online at www.ijrat.org}

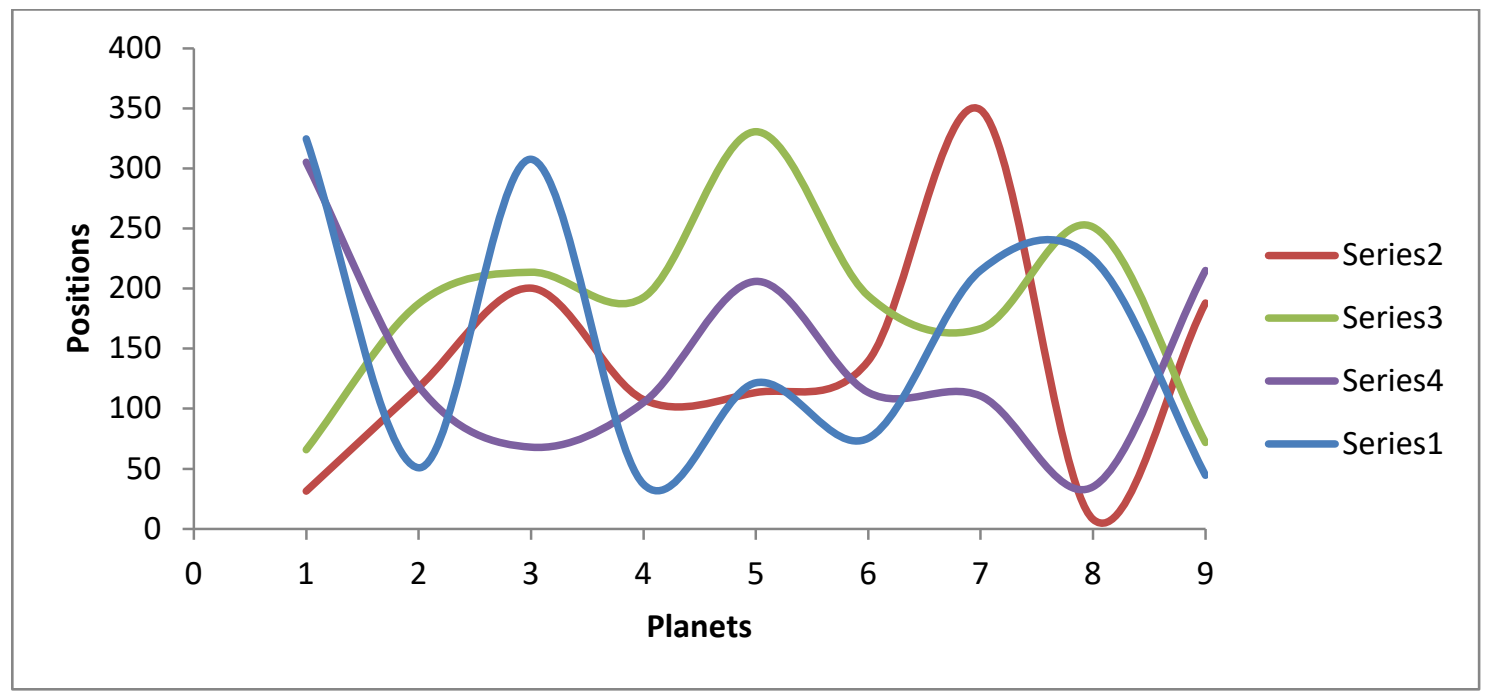

Figure 6: Brownian motion of different person's Horoscopes

From Fig 5 it is clear that the Random walk of the planets in the different person's Horoscope is entirely different and there is an irregular combination of planets induces the irregularity in the Random walk of each and every planets in each one's Horoscope these leads to the Fractal formation among the planets position and the Brownian motion of the planets in the Horoscope captures this irregularity noticeably as mentioned in Fig 6.

\section{CONSISTENCY USING THE POSITIONS OF THE PLANETS}

In a human's Horoscope, the position of the planets plays a vital role for prediction. Calculating Astrological Transits involves comparing the positions of the planets for any given time under consideration to the positions of the planets at the time of birth. Aspects between these Transiting planets and birth positions of the planets are noted as well as the Houses of the birth chart in which a Transiting planet would be passing through. With this planet's aspects it is possible to compare the Variability, Consistency, Stability, and Uniformity of two or more person's prospect. Basically in Statistics, Consistency of procedures, such as Computing Confidence intervals or Conducting Hypothesis tests is a desired property of their behaviour as the number of items in the data set to which they are applied increases indefinitely. Particularly, Consistency requires the outcome of the procedure with unlimited data should identify the underlying truth. The property of Consistency may be limited to one or more of the possible ways a sample size can grow. By considering the positions of the five major planets such as Jupiter, Saturn, Mercury, Mars and Moon in humans' Horoscope, each person's Stability in life can be judged. This will explained in detail with the following example.

Example: The following Table 2 gives the information about 5 planets positions in 2 different person's

Horoscope.

Table 2: Data for Analysis

\begin{tabular}{|c|c|c|c|c|c|}
\hline Planets & $\mathbf{1}$ & $\mathbf{2}$ & $\mathbf{3}$ & $\mathbf{4}$ & $\mathbf{5}$ \\
\hline Person A & 8 & 6 & 7 & 8 & 11 \\
\hline Person B & 2 & 8 & 7 & 9 & 9 \\
\hline
\end{tabular}

Compare the variability and state which person's Horoscope is more Consistent. 


\section{Available online at www.ijrat.org}

Table 3: Statistical values for Person A and Person B

\begin{tabular}{|c|c|c|c|c|c|c|}
\hline Planets & $\mathbf{1}$ & $\mathbf{2}$ & $\mathbf{3}$ & $\mathbf{4}$ & $\mathbf{5}$ & Total \\
\hline $\mathbf{X}$ & 8 & 6 & 7 & 8 & 11 & 40 \\
\hline $\mathbf{X}^{\mathbf{2}}$ & 64 & 36 & 49 & 64 & 121 & 334 \\
\hline $\mathbf{Y}$ & 2 & 8 & 7 & 9 & 9 & 35 \\
\hline $\mathbf{Y}^{2}$ & 4 & 64 & 49 & 81 & 81 & 279 \\
\hline
\end{tabular}

For the values in the above Table 3 the Coefficient of Variation can be determined by using the formula

$$
\text { Coefficient of Variation }=\frac{S . D}{\text { mean }} \times 100
$$

From eqn (8) the Coefficient of Variation for Person A is calculated as 21 and the Coefficient of Variation for Person B is calculated as 37.

Since the coefficient of variation for Person A is lesser, Person A is more Consistent than Person B. Hence the Person A's Horoscope is more Consistent than Person B's Horoscope.

The above example clearly states that the prediction of one's Horoscope is possible by the position of the planets in Horoscope, if the positions of the planets are more Consistent, the planets gives a favourable outcome throughout one's life time.

\section{CONCLUSION}

The approach of this paper is to illustrate human's Horoscope in the spotlight of Fractals based on Diffusion that process the combination of planets in the Horoscope with the characteristic of time scale that affects the transport of the planets and the Random walk of the planets induces the connection among the planets depends on time variation. This Diffusion process along with Random walk is an efficient way to model the changes in conjunction of the planets which is more efficient in analysing the Horoscope. Among the different methods utilized to analyze the human's Horoscope, calculating the Consistency of the planets in one's Horoscope results in more accurate prediction.

\section{ACKNOWLEDGEMENT}

The corresponding author expresses her profound gratitude and sincere thanks to the Management, VELS Institute of Science, Technology and Applied Studies (VISTAS), Chennai, Tamilnadu, India for their valuable support, permission and constant encouragement.

\section{REFERENCES}

[1] Svenson, S. \& White, K.A (1995), Content analysis of Horoscopes, Genetic, Social and General Psychology Monographs. 121, 1: 5-3.

[2] Ronny Martens and Tim Trachet, ( 1998), Making Sense of Astrology, Prometheus Books.

[3] Ove H. Sehested, (1973), The Basics Of Astrology, Volume 1, Uranus Publishing Co.

[4] Falconer K., (2014), Fractal Geometry, Mathematical Foundations and Applications, Third Edition.

[5] K. Falconer, (1997), Techniques in Fractal Geometry, Wiley, New York.

[6] M.T. Barlow, (1998), Diffusions on Fractals, Lecture Notes in Mathematics, Springer, Berlin, vol. 1690.

[7] R.Metzler, J. Klafter, (2000), The random walk's guide to anomalous diffusion: a fractional dynamic approach, Phys.Rep. 339 (1) 1-72.

[8] J.M. Angulo, M.D. Ruiz-Medina, V.V. Anh, W. Grecksch, (2000), Fractional diffusion and fractional heat equation, Adv. Appl. Probab. 32, 1077-1099.

[9] Bunde A, Havlin S, (1996), Fractals and disordered systems, ISBN 3-540-562192, Springer Verlag.

[10] Uthayakumar R, Jayalalitha G, (2009), Border detection of skin cancer cells with fractal dimension, Fractals 17, 171-180.

[11] S.P. Gupta, (2007), Statistical Methods, Sultan Chand \& Sons Publication, $35^{\text {th }}$ revised edition. 